\section{Atrial fibrillation is poorly tolerated by patients with hypertrophic concentric cardiomyopathy caused by mitochondrial tRNA ${ }^{\text {Leu (UUR) }}$ mutations}

\author{
Tiina M. Heliö, ${ }^{1}$ Alexandra Götz, ${ }^{2}$ \\ Janne Rapola, ${ }^{1}$ Sari Kiuru-Enari, 3,4 \\ Sari Kivistö, ${ }^{5}$ Terttu Heikinheimo, ${ }^{3}$ \\ Anu Suomalainen ${ }^{3,6}$ \\ 'Division of Cardiology, Helsinki \\ University Central Hospital, Heart and \\ Lung Center, Finland; ${ }^{2}$ Cornell University \\ Ithaca, NY, USA; ${ }^{3}$ Department of \\ Neurology, Helsinki University Central \\ Hospital, Finland; ${ }^{4}$ Department of Clinical \\ Neurosciences, University of Helsinki, \\ Finland; 5 Medical Imaging Center, Helsinki \\ University Central Hospital, Helsinki, \\ Finland; ${ }^{6}$ Research Programs Unit, \\ Molecular Neurology, Biomedicum- \\ Helsinki, University of Helsinki, Finland
}

\section{Abstract}

Knowledge on the molecular background of mitochondrial disorders has accumulated during the past two decades, but clinical and molecular features of mitochondrial cardiomyopathies (CMPs) are only starting to be characterized. We studied the detailed cardiologic phenotype of patients with adult-onset CMPs associated with mitochondrial DNA (mtDNA) mutations and their relatives, from three families. We identified a pathogenic $\mathrm{T} 3258 \mathrm{C}$ point mutation of mtDNA tRNA ${ }^{\text {Leu(UUR) }}$ in an adult patient with mitochondrial myopathy and CMP, with acute manifestation of dyspnea and elevated plasma $\mathrm{N}$-terminal pro-B-type natriuretic peptide concentration. Two other families with maternally segregated CMP, fatal in three patients, had the A3243G mutation in tRNA $^{\text {Leu(UUR) }}$ of mtDNA. Many of the mutation carriers, even if oligosymptomatic, had concentric, non-obstructive hypertrophic CMP with diastolic dysfunction or restrictive hemodynamics, or depression of systolic function especially at times when patients had lactic acidosis. Atrial fibrillation led to manifest heart failure in four patients, fatal in one. In conclusion, tRNA ${ }^{\text {Leu(UUR) }}$ mutations in mtDNA may underlie mitochondrial non-obstructive, sometimes fatal, hypertrophic CMPs of adult age. Such CMPs are characterized by left ventricular hypertrophy and poor tolerance of atrial arrhythmias, often leading to rapidly deteriorating systolic function and heart failure. These patients benefit of rapid intervention of tachyarrhythmias. These patients may not have neurological symptoms, and may therefore remain underdiagnosed. Our observations emphasize that patients with tRNA ${ }^{\text {Leu(UUR) }}$ mutations need cardiologic evaluation and follow-up. tRNA Leu(UUR) mutations should be screened of patients with hypertrophic CMP and metabolic acidosis, especially if atrial arrhythmia provoked cardiac decompensation.

\section{Introduction}

Mitochondrial DNA (mtDNA) mutations are a frequent cause of mitochondrial disease of adult age. Up to date, more than 120 pathogenic mtDNA point mutations have been described. ${ }^{1}$ MtDNA encodes 13 subunits of the respiratory chain (RC) enzymes, and its mutations cause RC deficiency and compromised oxidative ATP production. Therefore, tissues highly dependent on oxidative ATP-production, such as the skeletal muscle, heart, brain and neurosensory organs, are often affected by mtDNA mutations. However, mtDNA defects may manifest in a highly tissue specific manner, which cannot be explained by the mere ATP-deficiency. ${ }^{1,2}$

Recent reports suggest an important role for mitochondrial translation defects on early childhood cardiomyopathies (CMPs). ${ }^{3-5}$ However, molecular backgrounds of mitochondrial CMPs of adult age are still quite poorly known. Recessive ANT1 mutations were described to cause early-adulthood CMP in one pedigree ${ }^{6}$ and multiple mtDNA deletions were associated with CMP in a family with dominantly inherited dilated CMP. ${ }^{7}$ A3243G mutation in tRNA Leu(UUR) of mtDNA has been occasionally reported to include CMP as part of the disease manifestation..$^{8-13}$ This mutation typically manifests as mitochondrial myopathy and encephalopathy ${ }^{14}$ or as maternally inherited diabetes and deafness ${ }^{15}$ with high populationdependent frequency: from 1:420 in Australia to $\sim: 5000-1: 10,000$ in UK and Finland..$^{9,16,17}$

We report here the molecular genetics and presentation of mitochondrial myopathy with hypertrophic CMP in three families.

\section{Materials and Methods}

\section{Patients}

All the patient studies were done according to the Helsinki Declaration, with written informed consent from the patients. All subjects were of Finnish origin; Figure 1 illustrates their pedigrees. All individuals, whose mutation percentage is shown in the pedigree, were also clinically examined. Altogether 12 subjects underwent detailed neurologic and cardiologic examina-
Correspondence: Tiina M. Heliö, Department of Cardiology, Helsinki University Central Hospital, Meilahti Hospital, Haartmaninkatu 4, 00290 Helsinki, Finland.

Tel. +358.40.5819699 - Fax: +358.9.47176 652 .

E-mail: tiina.helio@hus.fi

Key words: mitochondrial DNA, respiratory chain deficiency, mitochondrial cardiomyopathy, T3258C, A3243G.

Funding: this study was supported by grants from Sigrid Juselius Foundation, Academy of Finland, Jane and Aatos Erkko Foundation, University of Helsinki (AS), Finnish Foundation for Cardiovascular Research (TMH), Finnish Medical Foundation (TMH) and from the special governmental subsidy for health sciences research, EVO-funds (AS and TMH), Finnish Cultural Foundation, Emil Aaltonen Foundation and Helsinki Graduate Program in Biotechnology and Molecular Biology (AG).

Acknowledgments: we acknowledge Dr. Anders Paetau for analyzing the muscle biopsies and Mrs. Sini Weckström for technical assistance.

Contributions: TMH, concept and design, patient material, analysis of cardiologic data, writing and editing of the manuscript and figures; AG, mitochondrial DNA analysis of patient 1 , mitochondrial DNA sequencing, mutation \% determination of the patients and the mother of patient 1 , blue native electrophoresis of patient 1 , writing and editing of the manuscript and figures; JR, analysis of cardiac data, writing and editing the manuscript; SK-E, patient examination, analysis of clinical data, editing of the manuscript and figures; SK, MRI analysis of patient, writing and editing of the manuscript and figures; TH, patient examination and analysis of clinical data (patient II:2, Family 1), writing and editing of the manuscript; AS, concept and design, patient material, analysis of data, writing and editing of the manuscript and figures. TMH, AS, contributed equally. All authors read and approved the final manuscript.

Conflict of interests: the authors declare no potential conflict of interests.

Received for publication: 15 November 2012

Revision received: 27 March 2013.

Accepted for publication: 17 May 2013.

This work is licensed under a Creative Commons Attribution NonCommercial 3.0 License (CC BYNC 3.0).

○Copyright T.M. Heliö et al., 2013

Licensee PAGEPress, Italy

Cardiogenetics 2013; 3:e6

doi:10.4081/cardiogenetics.2013.e6

tions and from two deceased patients data was obtained from hospital records. Table 1 summarizes their clinical findings. The age range of the subjects was 20-69 years and their body mass indexes varied between $18.1-26.4 \mathrm{~kg} / \mathrm{m}^{2}$. 


\section{Family 1}

The proband (II:2) is a 39-year old male, who presented at the age of 35 years with exercise-induced muscle pain, proximal leg weakness, dyspnea and sinus tachycardia 106/min and S3 in cardiac auscultation. He had been prescribed diuretics due to swelling of the ankles. Figure 2A shows the electrocardiogram (ECG). Serum pro-B-type natriuretic peptide (pro-BNP) concentration was elevated but thorax X-ray did not show pulmonary congestion. The main echocardiographic finding was nonobstructive left-ventricular hypertrophy (LVH; Figure 2A, Table 2). Doppler examination suggested elevated filling pressures. Magnetic resonance spectroscopy of the brain showed lactate increase in the thalamus, but no neurological signs or symptoms. Skeletal muscle sample showed that $20 \%$ of muscle fibers were cytochrome-c-oxidase (COX)-negative, succinate dehydrogenase positive, strongly supporting the diagnosis of mitochondrial dysfunction also underlying his cardiomyopathy. Family history was negative for mitochondrial diseases or cardiomyopathy. During clinical follow-up, his cardiologic condition has remained stable, without arrhythmias or dyspnea. Left ventricular walls have remained thickened, but the early peak diastolic LV/mitral annular velocity (E/Em) ratios have nearly normalized.

\section{Family 2}

The proband (III:6) is a woman with diabetes mellitus, sensorineural hearing impairment and depression. At the age of 51 years she developed acute pulmonary edema, hypertension and lactacidemia. Despite regular pulse and normal sized heart, serum troponin $\mathrm{T}$ and creatinine kinase-MB isoenzyme mass (CK-Mbm) levels were elevated (Table 1). ECG and echocardiography demonstrated $\mathrm{LVH}$, with diminished systolic function and restrictive hemodynamics (Figure 2B, Table 2). Coronary angiography demonstrated $70 \%$ stenosis in the proximal left anterior descending coronary artery (LAD), a total occlusion of middle LAD with collaterals from the right coronary artery, and $50 \%$ and $80 \%$ stenosis in first diagonal branch of LAD (D1) and second diagonal branch of LAD (D2), respectively. LV end diastolic pressure was $18 \mathrm{mmHg}$. Medical treatment of coronary artery disease was chosen. The diagnosis of mitochondrial contribution in hypertrophic CMP was strongly supported by histological results of skeletal muscle biopsy samples, showing over 30\% of COX-negative muscle fibers, many of them also showing the ragged-red fiber appearance, typical and diagnostic for mitochondrial myopathy. In followup she developed episodes of cardiac decompensation induced by atrial flutter (ventricular response of $90 / \mathrm{min})$ or fibrillation ( $110 / \mathrm{min})$ alternating with sinusbradycardia. These episodes were accompanied with metabolic

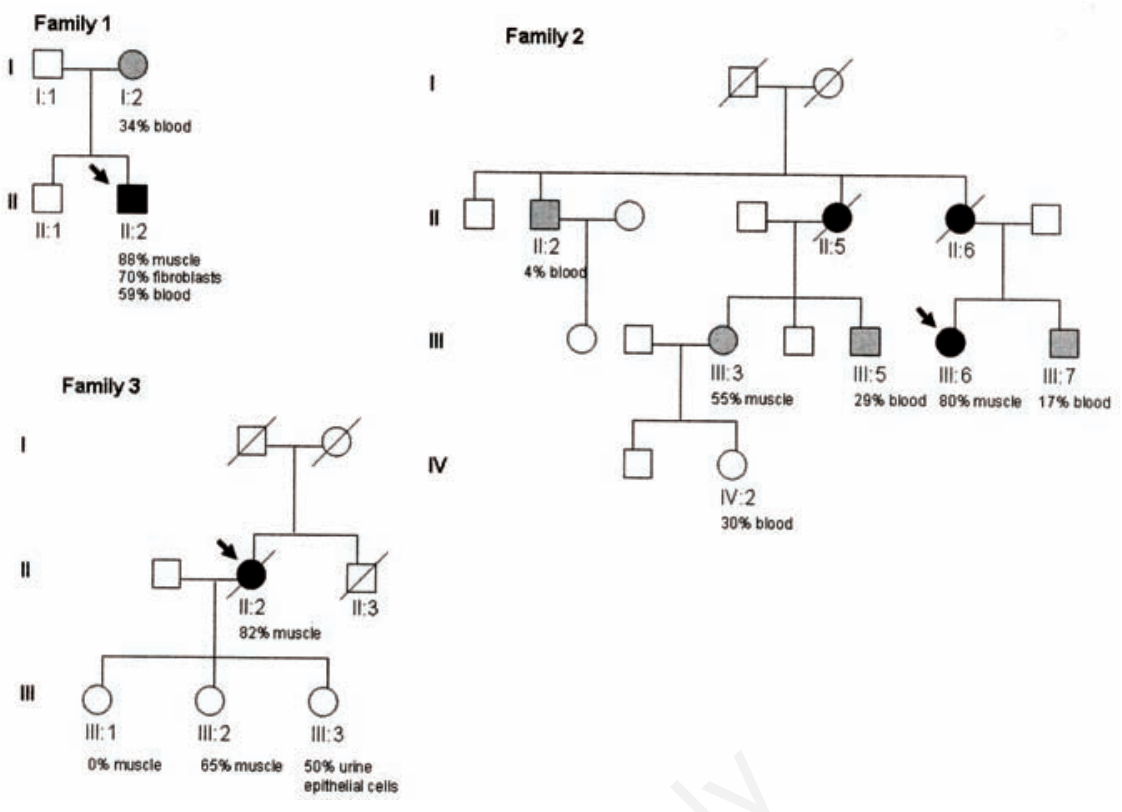

Figure 1. The family pedigrees. The index patients are marked with a black arrow. Black symbols indicate cardiac hypertrophy and signs or symptoms of heart failure; gray indicates subjects with increased relative wall thickness in echocardiography. MtDNA mutations were as followed: Family 1 - MELAS T3258C tRNA Leu(UUR); Family 2 and 3 MELAS A3243G tRNA Leu (UUR); \% indicates the people whose mutant mtDNA heteroplasmy levels were studied, and the amount of mutant mtDNA in different tissues.

A
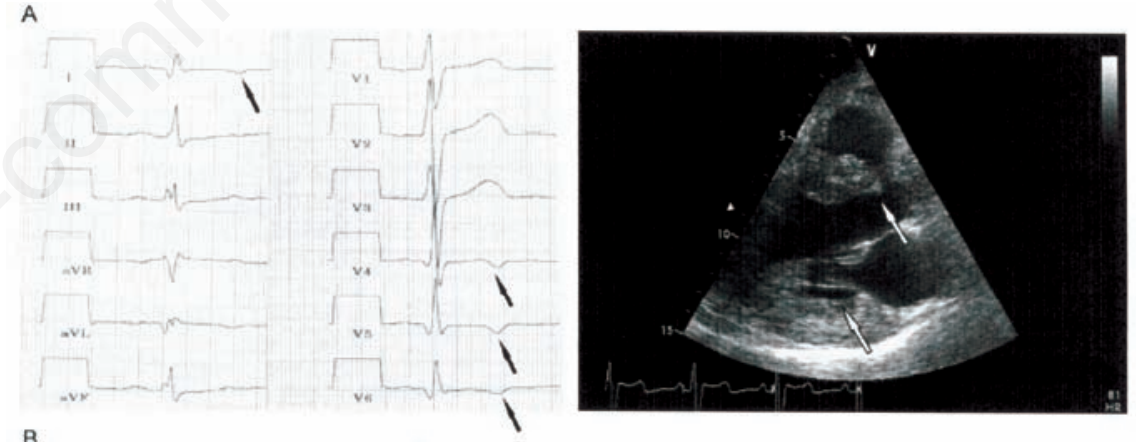

B

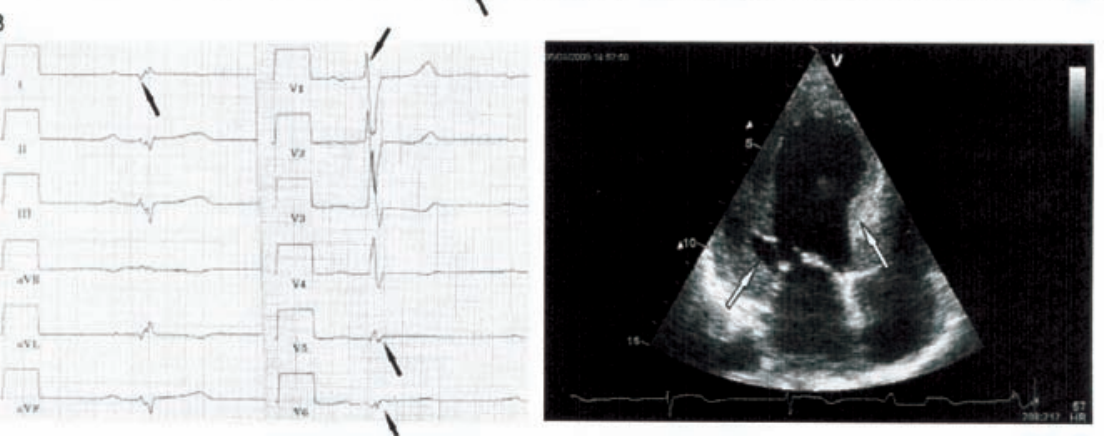

Figure 2. A) Patient II:2, family 1. Electrocardiogram (ECG) shows lateral t-inversions (left panel) and echocardiography (right panel) increased left ventricular wall thickness in parasternal long axis view (arrows); B) Patient III:6, family 2. ECG shows lateral q-waves and abnormal r-peaks in the lateral chest leads (left panel), echocardiography (right panel) left ventricular hypertrophy (arrows). Apical view. 
acidosis. Hemodynamics improved and lactacidemia subsided after sinus rhythm was restored. Plasma N-terminal pro-B-type natriuretic peptide (NT-proBNP) concentration was high, up to $25,505 \mathrm{ng} / \mathrm{L}$, but decreased upon compensation. She received a physiologic pacemaker. Patient II:5 was a woman who at 66 years of age had an episode of cardiac decompensation with atrial fibrillation and left bun- dle branch block, but no ventricular tachycardia. On echocardiography LV ejection fraction was $40 \%$. She had mild renal failure and serum NT-proBNP concentration was high (Table 1). Brain computed tomography revealed old infarctions in both hemispheres and in cerebellum; she was diagnosed with multi-infarction dementia. Coronary angiography had been carried out at the age of 65 years, it had revealed one significant stenosis of the left coronary circumflex branch that was treated by percutaneous coronary intervention and stenting. At 68 years, she was hospitalized because of chest pain; her blood pressure was $95 / 60$ $\mathrm{mmHg}$, she had sinus tachycardia (113/min), and an enlarged heart with pulmonary venous congestion. Echocardiography was not performed. The patient succumbed soon there-

Table 1. Clinical findings of the subjects.

\begin{tabular}{|c|c|c|c|c|c|c|c|c|c|c|c|c|c|c|}
\hline Parameter, ref. values & & mily 1 & & & & mily 2 & & & & & & & ly 3 & \\
\hline Patient & $\mathrm{I}: 2$ & II:2 & II:2 & II: 5 & II:6 & III:3 & III:5 & III:6 & III:7 & $\mathrm{IV}: 2$ & II:2 & III:1 & III:2 & III:3 \\
\hline Gender & $\mathrm{F}$ & $\mathrm{M}$ & M & $\mathrm{F}$ & $\mathrm{F}$ & $\mathrm{F}$ & M & $\mathrm{F}$ & M & $\mathrm{F}$ & $\mathrm{F}$ & $\mathrm{F}$ & $\mathrm{F}$ & $\mathrm{F}$ \\
\hline Age (years) & 69 & 39 & 69 & 68 & 66 & 51 & 41 & 57 & 52 & 20 & 58 & 27 & 34 & 36 \\
\hline Height (cm) & 164 & 185 & 180 & 160 & 156 & 163 & 167 & 161 & 171 & 168 & 157 & 163 & 162 & 165 \\
\hline Weight (kg) & 71 & 63 & 64 & 46 & 44 & 60 & 65 & 47 & 73 & 60 & 47 & 55 & 60 & 69 \\
\hline Hypertension & + & - & - & + & - & - & + & + & + & - & + & - & - & - \\
\hline CAD in angio-graphy or autopsy & $\mathrm{NA}$ & $\mathrm{NA}$ & $\mathrm{NA}$ & + & - & $\mathrm{NA}$ & $\mathrm{NA}$ & + & $\mathrm{NA}$ & NA & - & $\mathrm{NA}$ & $\mathrm{NA}$ & $\mathrm{NA}$ \\
\hline Heart failure & - & + & - & + & + & - & - & + & - & - & + & - & - & - \\
\hline Diabetes & - & - & - & + & - & + & + & + & - & - & + & - & - & - \\
\hline Hearing loss & - & - & - & + & + & + & + & + & + & - & + & - & - & - \\
\hline Depression & - & - & - & - & + & - & - & + & + & - & - & - & - & - \\
\hline Fatigue and/or anorexia & + & - & - & + & + & - & - & + & + & - & + & - & - & - \\
\hline Dyspnea & - & + & - & + & - & - & - & + & - & - & + & - & - & - \\
\hline Chest pain & + & - & + & + & - & - & - & + & - & - & + & - & - & - \\
\hline Palpitation & + & + & + & $\mathrm{NA}$ & - & - & - & + & + & - & + & - & - & - \\
\hline $\begin{array}{l}\text { Age at onset of any } \\
\text { cardiac symptoms (years) }\end{array}$ & 52 & 35 & $\sim 50$ & 56 & 66 & - & - & 51 & 52 & - & 56 & - & - & - \\
\hline Systolic RR mmHg & 150 & 129 & 149 & 95 & 130 & 118 & 144 & 150 & 147 & 121 & 143 & 114 & 109 & 110 \\
\hline Diastolic RR mmHg & 90 & 68 & 82 & 60 & 90 & 77 & 99 & 100 & 104 & 75 & 109 & 85 & 78 & 77 \\
\hline Abnormal auscultation & - & S3 & - & $\begin{array}{l}\text { Systolic } \\
\text { murmur }\end{array}$ & - & - & - & - & - & - & - & - & - & - \\
\hline NT-proBNP (ng/L)* & - & 345 & - & 16,159 & $\mathrm{NA}$ & - & 117 & 14,126 & - & - & 27,382 & $\mathrm{NA}$ & $\mathrm{NA}$ & $\mathrm{NA}$ \\
\hline $\mathrm{CK}(\mathrm{U} / \mathrm{L})^{\circ}$ & - & 988 & - & 214 & $\mathrm{NA}$ & - & - & 1064 & - & - & - & - & $\mathrm{NA}$ & - \\
\hline CKMBm $(\mu \mathrm{g} / \mathrm{L})^{\#}$ & 3 & 75 & $\mathrm{NA}$ & 22 & 31 & $\mathrm{NA}$ & $\mathrm{NA}$ & 25 & $\mathrm{NA}$ & $\mathrm{NA}$ & 14 & $\mathrm{NA}$ & $\mathrm{NA}$ & $\mathrm{NA}$ \\
\hline $\operatorname{TnT}(\mu \mathrm{g} / \mathrm{L})^{\S}$ & $\mathrm{NA}$ & 0.09 & NA & 0.16 & $\mathrm{NA}$ & $\mathrm{NA}$ & $\mathrm{NA}$ & 0.17 & $\mathrm{NA}$ & $\mathrm{NA}$ & 0.07 & $\mathrm{NA}$ & $\mathrm{NA}$ & $\mathrm{NA}$ \\
\hline Lactate $(\mathrm{mmo} / \mathrm{L})^{\wedge}$ & 0.9 & 5.3 & 0.9 & NA & $\mathrm{NA}$ & 0.9 & 2.2 & 7 & 2.4 & 1.2 & 5.1 & 1 & 1.5 & 1.2 \\
\hline Abnormal ECG & - & + & + & + & + & - & - & + & + & - & + & - & - & + \\
\hline AV-block & - & - & + & - & + & - & - & - & + & - & - & - & - & - \\
\hline LVH & - & + & - & - & - & - & - & + & - & - & + & - & - & - \\
\hline LAH & - & Transient & - & - & - & - & - & - & - & - & + & - & - & - \\
\hline LBBB & - & - & - & + & - & - & - & - & - & - & - & - & - & - \\
\hline q-waves & - & + & - & - & - & - & - & + & - & - & $\begin{array}{l}\text { Antero- } \\
\text { septal }\end{array}$ & - & - & - \\
\hline Atrial fibrillation or flutter & $\begin{array}{c}\text { prx in } \\
24 \mathrm{~h}-\mathrm{ECG}\end{array}$ & - & $\begin{array}{c}\text { Short prx } \\
\text { in } 24 \mathrm{~h} \\
\text { ECG }\end{array}$ & prx & - & - & - & prx & - & - & + & - & - & - \\
\hline
\end{tabular}

Sinustachycardia

Other ECG changes

Inferolateral

$+\quad-\quad-$

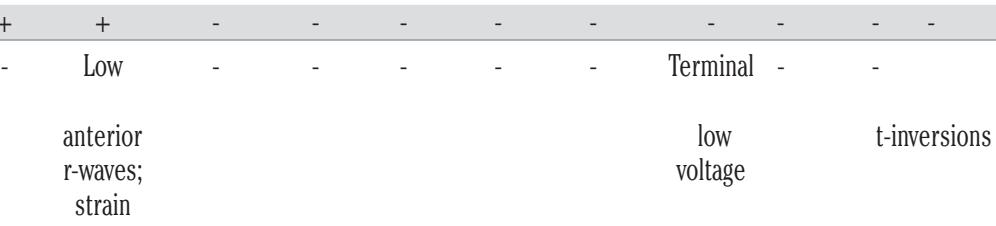

Age at the last examination is reported. Abbreviations: CAD, coronary artery disease; NA, not available; RR, blood pressure; NT-proBNP, N-terminal pro B-type natriuretic peptide; CK, creatinine kinase; CKMBm, CK-MB isoenzyme mass; TnT, troponine T subunit; ECG, electrocardiography; AV, atrioventricular; LVH, left ventricular hypertrophy; LAH, left atrial hypertrophy; LBBB, left bundle branch block; prx, paroxysmal, paroxysms; \%b, mutation \% in blood; \%m, mutation \% in skeletal muscle; \%uec, mutation \% in urinary epithelial cells. *Normal range for NT-proBNP (ng/L): F $<50$ y<155 ng/L, F $50-65$ y<222 ng/L, F $>65$ y<352 ng/L, M<50 y<84 ng/L, M $50-65$ y<194 ng/L, 
after. Autopsy showed cardiac hypertrophy and diffuse coronary atherosclerosis. Light microscopic analysis showed myocardial fibrosis, compatible with CMP. Patient II:6 was a woman, who at 65 years of age was admitted to hospital because of fatigue and anorexia. She had sensorineural hearing loss, and memory difficulties, but no dementia. ECG showed strain, and she became hypotensive and hypoxemic, with metabolic acidosis, hyponatremia, severe hyperkalemia, pulmonary venous congestion and pleural effusion. Echocardiography was not performed. Despite intensive treatment, hypotension persisted, and the patient died of asystole at the age of 66 years. Autopsy revealed LVH. Light microscopy of the myocardium showed fibrosis, lymphocyte infiltration and disarray of fibers, indicative for CMP. The coronaries were open.

\section{Family 3}

The proband (II:2). At 56 years of age this short and lean woman with asthma and hearing impairment (since 33 years of age) had her first episode of atrial fibrillation. She was slightly hypertensive, ECG showed strain and pulse rate of $64 / \mathrm{min}$, and CK-MBm, troponin T and creatinine concentrations were slightly elevated (Table 1). Echocardiography showed LVH, with ejection fraction (EF) of $46 \%$ and moderate mitral and tricuspid regurgitation (Table 2). Coronary angiogram showed open coronaries. Endomyocardial biopsy showed nuclear hypertrophy. Right heart catheterization demonstrated a mean wedge pressure of $17 \mathrm{mmHg}$, pulmonary artery systolic pressure of 47 $\mathrm{mmHg}$ and cardiac output of $3.3 \mathrm{~L} / \mathrm{min}$. She was diagnosed with type- 2 diabetes. At 58 years of age she developed dyspnea and pleural effusion with atrial fibrillation (110/min). Elective cardioversion returned sinus rhythm only transiently. Echocardiography showed severe left-ventricular dysfunction, with wall thickness of $15-16 \mathrm{~mm}$ and EF $20-29 \%$. She became rapidly hypotensive, hypoxic, developed deep metabolic acidosis and low voltage ECG. Despite intensive care, she succumbed in cardiogenic shock. At autopsy, mitochondrial disease was diagnosed, as $\sim 20 \%$ of the skeletal muscle fibers were COX-negative. She had a hypertrophic heart, with both chambers and right atrium dilated, and pericardial effusion. Light microscopic examination demonstrated remarkable myocardial fibrosis, consistent with CMP. Coronaries were open.

\section{Echocardiography and cardiac magnetic resonance imaging}

Original digital echocardiographic data were available in 10 out of 12 cases. In two cases (Family 1, I:2; Family 3, II:2) data were obtained from medical records. We regarded left ventricular wall thickness exceeding 10 mm abnormal. Relative wall thickness (RWT) was calculated by the formula (2XPWTd)/ LVIDd. RWT $>0.42$ was considered abnormal. Of the tissue Doppler velocities, mitral inflow $\mathrm{E}$ wave $>1 \mathrm{~m} / \mathrm{s}$ and $\mathrm{E} / \mathrm{Em}>15$ were considered indicative for restrictive hemodynamics. Cardiac magnetic resonance imaging (MRI) was performed with 1.5 T MR imager (Avanto, Siemens, Erlangen, Germany). Breath-hold cine images were performed using retrospectively gated segmented true fast imaging with steady-state free precession techniques. Cine
MR images were obtained in 4-chamber, 3chamber and short-axis planes covering both ventricles. Delayed enhancement images were acquired 15 min after injection of contrast agent (gadoterate meglumine $0.1 \mathrm{mmol} / \mathrm{kg}$ ) in the same views as for cine images, using inversion recovery turbo fast-low angle shot. Inversion time was optimized for each measurement to null the signal intensity of normal myocardium (240-360 ms). Cine images were analyzed with Argus application (Siemens) developed for this purpose. Delayed enhanced images were analyzed visually.

\section{mtDNA analyses}

Total DNA from snap-frozen muscle tissue, blood samples or cultured fibroblasts was extracted by phenol-chlorophorm and precipitated with ethanol, according to standard methods. The full mtDNA sequence (for index patient II:2 of family 1), was determined by high-throughput sequencing as described by Pietiläinen et al. ${ }^{18}$ using revised Cambridge sequence of complete human mtDNA (NC_012920.1) as reference. Sequence processing and analysis was done with Sequencher 4.5 software (GeneCodes Corp., Ann Arbor, MI, USA). For mutant T3258C mtDNA quantification and for A3243G mutant detection and quantification we utilized solidphase minisequencing, as studied by Suomalainen and Syvänen. ${ }^{19}$ The primer sequences for T3258C were as follows:

F: 5' Biotin-ACCCACCCAAGAACAGGGTTTGTTA 3' R: 5' CATGGGTATGTTGTTAAGAAGAGGAATTGAACCTC 3' R-detection: 5' ACTGTAAAGTTTTAAGTTTT 3'.

Table 2. Echocardiography findings in study participants.

\begin{tabular}{|c|c|c|c|c|c|c|c|c|c|c|c|c|c|}
\hline Family & 1 & 1 & 2 & 2 & 2 & 2 & 2 & 2 & 3 & 3 & 3 & 3 & Ref. value \\
\hline Patient & $\mathrm{I}: 2^{*}$ & $\mathrm{II}: 2$ & II:2 & III:3 & III:5 & III:6 & III:7 & IV:2 & $\mathrm{II}: 2^{*}$ & III:1 & III:2 & III:3 & - \\
\hline Age & 69 & 39 & 69 & 51 & 41 & 57 & 52 & 20 & 58 & 27 & 34 & 36 & - \\
\hline LVEDD (mm) & 45 & 53 & 49 & 45 & 44 & 49 & 46 & 40 & 47 & 47 & 46 & 54 & $\begin{array}{l}<54(\mathrm{~F}) \\
<60(\mathrm{M})\end{array}$ \\
\hline EF (\%) & 66 & 61 & 61 & 62 & 61 & 52 & 58 & 60 & 46 & 58 & 56 & 56 & $>55 \%$ \\
\hline FS (\%) & $\mathrm{NA}$ & 30 & 33 & 30 & 32 & 24 & 43 & 30 & NA & 34 & 35 & 33 & $>25 \%$ \\
\hline Septum (mm) & 11 & 14 & 13 & 12 & 14 & 23 & 20 & 10 & 15 & 7 & 8 & 8 & $\begin{array}{l}<10(\mathrm{~F}) \\
<11(\mathrm{M})\end{array}$ \\
\hline Posterior wall (mm) & 12 & 12 & 10 & 10 & 12 & 20 & 13 & 10 & 14 & 7 & 7 & 8 & $\begin{array}{l}<10(\mathrm{~F}) \\
<11(\mathrm{M})\end{array}$ \\
\hline RWT (cm) & 0.53 & 0.45 & 0.41 & 0.44 & 0.55 & 0.57 & 0.57 & 0.50 & 0.78 & 0.30 & 0.35 & 0.30 & $<0.42$ \\
\hline $\mathrm{E} / \mathrm{Em}$ & NA & 9.6 & 8.6 & NA & $\mathrm{NA}$ & 35 & 9.8 & 4.5 & NA & 5.7 & 4.9 & 4.8 & $<8^{\circ}$ \\
\hline $\mathrm{E}(\mathrm{m} / \mathrm{s})$ & NA & 0.77 & 0.6 & 0.6 & $\mathrm{NA}$ & 1.05 & 0.49 & 0.9 & 0.6 & 0.85 & 0.83 & 0.62 & $<1.0^{\#}$ \\
\hline $\mathrm{A}(\mathrm{m} / \mathrm{s})$ & NA & 0.51 & 0.84 & 0.6 & $\mathrm{NA}$ & 0.42 & 0.59 & 0.4 & $\mathrm{NA}$ & 0.32 & 0.41 & 0.34 & $\S$ \\
\hline $\operatorname{Em}(\mathrm{m} / \mathrm{s})$ & NA & 0.08 & 0.07 & NA & 0.12 & 0.03 & 0.05 & 0.2 & $\mathrm{NA}$ & 0.15 & 0.17 & 0.13 & $>0.10^{\#}$ \\
\hline $\operatorname{Am}(\mathrm{m} / \mathrm{s})$ & NA & 0.09 & 0.11 & $\mathrm{NA}$ & 0.05 & 0.02 & 0.07 & 0.08 & $\mathrm{NA}$ & 0.04 & 0.05 & 0.09 & $\S$ \\
\hline
\end{tabular}

From two individuals $\left({ }^{*}\right.$ ) only written description of echocardiographic findings was available, others were analyzed by single investigator (J.R.); ${ }^{\circ}$ ratio of $<8$ suggests normal filling pressures and diastolic function. Values above 15 suggest elevated filling pressures, intermediate values 8-15 represent a grey zone; "values depend on age, sex and volume status. An E velocity of $>1 \mathrm{~m} / \mathrm{s}$ and an Em value of less than $10 \mathrm{~cm} / \mathrm{s}$ are usually considered abnormal; ${ }^{8}$ no reference values exist for A and Am. LVEDD, left ventricular end diastolic dimension; EF, ejection fraction; FS, fractional shortening; RWT, relative wall thickness; E, early peak diastolic LV filling; Em, early peak diastolic mitral annular velocity; A, late (atrial) diastolic LV filling; Am, late (atrial) diastolic mitral annular velocity. None of the subjects had a significant valvular defect except for the proband of family 3 (II:2), who had significant tricuspid valve regurgitation and an enlargened right atrium. Abnormal values are in italics. 


\section{Respiratory chain protein analysis of fibroblasts}

Primary fibroblast cell lines of patient II:2 were cultured according to standard conditions in $5 \% \mathrm{CO}_{2}$, Dulbecco modified Eagle medium supplemented with $10 \%$ fetal bovine serum. Mitochondrial membranes with intact respiratory chain complexes were extracted from a total of about four million cells as previously described.$^{20}$ The samples were separated on a 6 $15 \%$ blue native polyacrylamide gel electrophore$\operatorname{sis}^{21}$ and blotted onto a polyvinylidene fluoride membrane (EMD Millipore Corp., Billerica, MA, USA). For protein detection, we used monoclonal antibodies (Mitosciences, Eugene, OR, USA) against the $39 \mathrm{kDa}$ subunit of Complex I, and 70 $\mathrm{kDa}$-Ip subunit of Complex II. In-gel histochemical Complex I holoenzyme activity of the patient's fibroblasts was measured after blue native electrophoresis, as previously described. ${ }^{22}$ Complex I activity was quantified by calculating the band intensity against the protein amount of Complex II, in a parallel gel.

\section{Results}

\section{DNA analysis}

MtDNA sequence analysis of patient II:2 from family 1 showed a heteroplasmic $\mathrm{T}$ to $\mathrm{C}$ transition at the highly conserved nucleotide position 3258 within the mitochondrial tRNA Leu (UUR) gene. No other heteroplasmic or potentially pathogenic variants were found in the analysis (variants excluded as pathogenic, if present in the mitochondrial polymorphic variant databases in over $1 \%$ of individuals; www.mitomap.org, www.mtdb.igb.uu.se). The proportion of the mutant mtDNA was $88 \%$ of total mtDNA in the skeletal muscle, $70 \%$ in the fibroblasts and $59 \%$ in blood. The mother of the proband (I:2) also carried the heteroplasmic mutation, in a lower amount of $34 \%$ of total mtDNA in her blood, confirming maternal inheritance. In families 2 and 3 , the index patients and their maternal relatives had the A3243G transition within tRNA Leu (UUR) of mtDNA in variable amounts (Figure 1, Table 1). Both T3258C and A3243G mutations have previously been proven to be unequivocally pathogenic, based on both genetic and biochemical data:;4,23,24 mutant mtDNA amounts were lower in blood than in skeletal muscle, which is a known feature of A3243G mutant mtDNA: progressive loss of mutant mtDNA from blood occurs during aging. ${ }^{25}$

\section{An overview of the cardiac findings}

Table 1 summarizes the clinical findings and Table 2 the echocardiographic findings. Of 14 individuals with mtDNA mutations or maternally related likely carriers, eight had cardiac symptoms, mainly palpitations, chest pain and dyspnea. Five subjects had paroxysmal or chronic atrial fibrillation. None had remarkable atrioventricular conduction disturbances or documented ventricular arrhythmias. Two patients had ECG changes compatible with LVH.

Echocardiography showed normal sized left ventricles, but thickened left ventricular walls (>10 mm) in eight subjects, aged $39-69$ years (mean 54.5 years), whereas those with normal left ventricular wall thickness were younger, aged 20-36 years (mean 24.8). Relative wall thickness was increased in $8 / 12$ (67\%) subjects, aged 20-69 years (mean 48.4). Those with normal RWT were aged 27-69 years (mean 41.5). None of the subjects had left ventricular outflow tract obstruction. Prominent LVH was associated with diastolic dysfunction, even restrictive hemodynamics. The systolic function was mainly normal at rest.

Three female patients, aged 58-68 years, died of rapidly developing heart failure. Two patients had sinustachycardia and one atrial fibrillation in terminal phase. In addition, one subject has had several episodes of acute heart failure precipitated by atrial fibrillation. The tachyarrhythmias typically associated with lactic acidosis and rapidly rising plasma NTproBNP level. The response to inotropes was poor.

Cardiac MRI of patient II:2, family 1, demon-

\section{A}
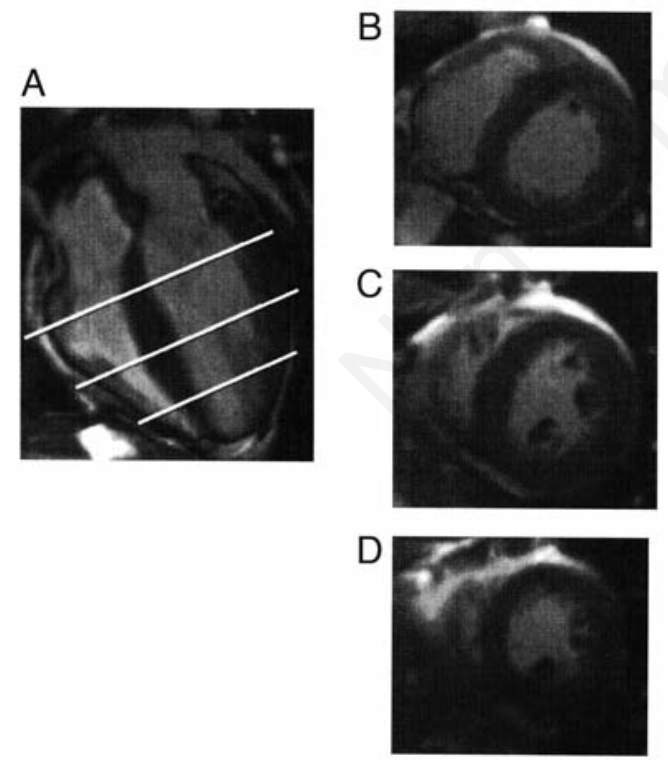

E

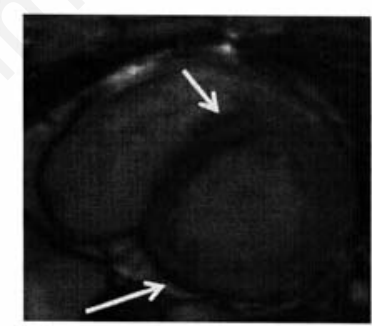

F

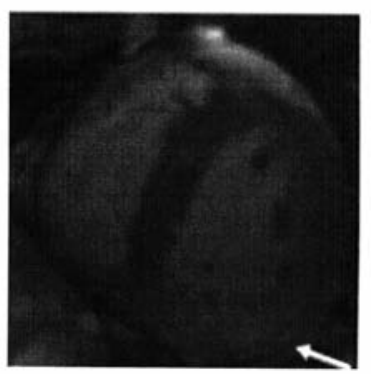

Figure 3. Cardiac magnetic resonance imaging findings of patient II:2, family 1, a 36-year old male patient: (A) four-chamber view (B) basal short-axis view, (C) mid-ventricular short-axis view, (D) axial short-axis view. Left ventricular (LV) volumetric measurements of determined from short-axis cine images: end-diastolic volume $167 \mathrm{~mL}\left(92 \mathrm{~mL} / \mathrm{m}^{2}\right)$, LV end-systolic volume $55 \mathrm{~mL}\left(31 \mathrm{~mL} / \mathrm{m}^{2}\right)$, stroke volume $112 \mathrm{~mL}\left(62 / \mathrm{m}^{2}\right)$, ejection fraction $67 \%$, mass $250 \mathrm{~g}$ $\left(139 \mathrm{~mL} / \mathrm{m}^{2}\right)$. Ventricular thickness was increased mostly in basal and mid-ventricular parts. Anteroseptal wall thickness was maximally $20 \mathrm{~mm}$ and posterolateral wall thickness maximally $18 \mathrm{~mm}$. (E) Delayed enhanced short-axis images in basal and (F) mid-ventricular parts of left ventricle. Diffuse (=greyish) patchy transmural enhancement was found mainly from basal anteroseptum and posterolateral wall (arrows).
A

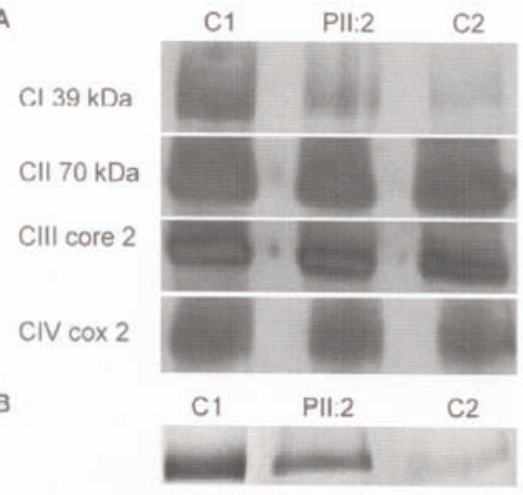

Figure 4. A) Mitochondrial respiratory chain analysis of fibroblasts: blue-native polyacrylamide-gel electrophoresis of mitochondrial protein preparations. PII:2, patient II:2 from family 1 , with cardiomyopathy caused by mtDNA T3258C mutation. Decreased amount of fully assembled complex I. C1: healthy subject fibroblasts; C2: Complex I deficient patient sample with $\mathrm{T} 3271 \mathrm{C}$ point mutation in mtDNA. CI, CII, CIII, CIV; respiratory chain complexes I-IV. $39 \mathrm{kDa}, 70$ $\mathrm{kDa}$, core- 2 and cox- 2 indicate against which complex subunits the used antibodies were detecting. B) in gel enzymatic activity assay for NADH ubiquinone oxidoreductase activity of Complex I. 
strated preserved end-diastolic and -systolic volumes and functions, irregularly thickened left ventricular walls and increased global mass of the heart ${ }^{26,27}$ (Figure 3).

Six subjects had hypertension, five had diabetes and two had coronary artery disease verified by angiography or autopsy.

\section{Respiratory chain protein analysis}

Blue native gel electrophoresis, separating respiratory chain complexes as whole functional enzyme complexes in native gel, indicated a decreased amount of fully assembled complex I (containing seven mtDNA-encoded subunits, the translation of which is dependent on functional mitochondrial tRNAs), when compared to Complex II (fully encoded by nuclear genes, and therefore unaffected by mitochondrial DNA defects). Furthermore, in-gel Complex-I enzyme activity assay in the native gel showed of marked Complex-I deficiency in the patient with T3258C mutation (Figure 4). This result supported mitochondrial respiratory chain dysfunction caused by $\mathrm{T} 3258 \mathrm{C}$ mutant mtDNA.

\section{Discussion}

We report here maternally inherited mitochondrial tRNA Leu (UUR) mutations A3243G and T3258C in patients with adult-onset hypertrophic CMP and show that their oligosymptomatic mutation-carrying relatives also often have cardiac manifestations. Hypertrophic CMP was the main manifestation of the disease in our three families. We show that atrial arrhythmias in these patients may lead to rapidly progressive cardiac insufficiency, lethal in some of our patients. The A3243G change is the most commonly found mtDNA mutation, originally described to cause mitochondrial encephalopathy, lactic acidosis and stroke-like episodes (MELAS), but most often manifesting as maternally inherited diabetes and deafness. ${ }^{14,15}$ Typical for A3243G disorders is the wide variation in clinical manifestations, but only few case reports of CMP as a major presenting manifestation exist. ${ }^{8-13}$ We conclude that cardiac manifestations associated to A3243G and T3258C mutations, despite the common occurrence of the former, ${ }^{9,16,17}$ are not well known among physicians. If diagnosed early, the malignant disease course of these mitochondrial CMPs upon atrial fibrillation could be affected by prompt intervention.

The heteroplasmic T3258C tRNA Leu (UUR) mutation of mtDNA has previously been described to cause MELAS, similar to A3243G mutation, but no CMP. ${ }^{23,28}$ However, the clear finding of mitochondrial myopathy in the patients' muscle and Complex-I deficiency in fibroblasts indicate that the T3258C-disease affects several tissue types, and is likely to underlie the cardiac symptoms as well in the patient. The clinical manifestations associated with tRNA mutations of mtDNA are typically extremely variable. ${ }^{1}$ The cardiac manifestations co-segregating in the large pedigrees

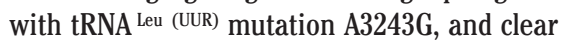
findings of mitochondrial myopathy in the muscle also strongly support the causative role of this mutation underlying CMP of our patients. Furthermore, the number of reported patients and biochemical evidence have clearly established the pathogenic role of both T3258C and A3243G: they lead to tRNA Leu (UUR) dysfunction, deficient mitochondrial protein translation and respiratory chain deficiency. ${ }^{23}$ Our findings show that mtDNA mutations should be considered as possible causes of hypertrophic concentric CMPs, even if neurological or muscle symptoms were not present.

Previous studies have suggested that the risk of developing $\mathrm{LVH}$ is related to the general severity of the phenotype of the mitochondrial disease and to a lesser degree to age. ${ }^{13}$ However, in our patients, LVH was the leading manifestation of the disease, progressive by age. Tachycardia, especially atrial fibrillation or flutter led rapidly to deterioration of the left ventricular systolic function and florid, sometimes fatal heart failure, associated with metabolic acidosis. Hypertrophic CMP caused by sarcomeric mutations, with substantial left ventricular wall thickening, lead often to ventricular tachyarrhythmia or left ventricular outflow tract obstruction. None of our patients had documented ventricular tachyarrythmias or obstruction, or atrioventricular conduction abnormality, although their left ventricular wall thickness was comparable to that in typical sarcomeric CMPs. End-stage CMP in our patients was associated with fibrosis, indicating cardiomyocyte degeneration. Our results show that mitochondrial CMP patients are particularly vulnerable to tachycardia, and that imminent intervention for atrial tachycardias is important.

Typical mitochondrial CMPs are nonobstructive and concentric, ${ }^{11,13,29}$ but also dilated CMP has been described..$^{30,31}$ In our patients, the decrease of left ventricular systolic function was not associated with an increased left ventricular end diastolic dimension, but instead, the left ventricular walls were thickened, especially upon acute decompensation. The cardiac MRI showed diffuse mild intensity enhancement, suggestive of edema, in hypertrophic areas, mainly in basal anteroseptal and posterolateral wall. MRI findings in hypertrophic sarcomeric CMPs typically show delayed mid-myocardial enhancement in hypertrophic parts of septum and at the junctions of interventricular septum and right ventricle. $^{32}$ In Anderson-Fabry CMP enhancement locates predominantly in the posterolateral wall. In sarcoidosis thickening and enhancement of ventricular wall can be found in most cases in the same area as in our patient, basal septum and laterobasal wall, but typically patchy and nodular mid-myocardial and subepicardial areas exist. ${ }^{33}$ These findings suggest that different molecular causes of hypertrophic CMP may affect different regions of myocardium.

Both T3258C and A3243G mutations in tRNA $^{\text {Leu(UUR) }}$ lack the normal taurine-containing modification (5-taurinomethyluridine) at the anticodon wobble position, leading to inefficient and imprecise codon recognition and aminoacylation. ${ }^{23}$ The defective mitochondrial translation, due to lack of leucine incorporation and misincorporation of phenylalanine in place of leucine to proteins, ${ }^{34}$ is supported by the complex-I deficiency in our T3258C patient cells. Complex I contains the highest amount of leucine, and has the highest number of mtDNA-encoded subunits. Accordingly, complex I deficiency has been reported to be typical for A3243G and A3260G mutation in tRNA Leu (UUR), the latter also associated with mitochondrial CMP. ${ }^{35-37}$ Occasional CMP patients with tRNA ${ }^{\text {lle }}$, tRNA ${ }^{\text {Gly }}$ and tRNA Leu (CUN) mutations have been reported. ${ }^{35,38-40}$ Why CMP is not, however, a common consequence of all mitochondrial tRNA mutations or not even always a manifestation of A3243G in all families remains unsolved.

Our study shows that patients with tRNA ${ }^{\text {Leu(UUR) }}$ mutations need cardiologic evaluation and follow-up. The triad of diastolic dysfunction, atrial arrhythmias and metabolic acidosis may be life-threatening for these patients, and should be recognized especially in an acute setting: prompt treatment of tachycardia might prevent development of irreversible heart failure in mitochondrial disease patients. Furthermore, tRNA ${ }^{\text {Leu(UUR) }}$ mutations should be screened in patients with hypertrophic CMP and metabolic acidosis, especially, if atrial arrhythmia provoked cardiac decompensation.

\section{References}

1. Ylikallio E, Suomalainen A. Mechanisms of mitochondrial diseases. Ann Med 2011;44: 41-59.

2. Nunnari J, Suomalainen A. Mitochondria: in sickness and in health. Cell 2012;148: 114559.

3. Götz A, Tyynismaa H, Euro L, et al. Exome sequencing identifies mitochondrial alanyltRNA synthetase mutations in infantile mitochondrial cardiomyopathy. Am J Hum Genet 2011;88:635-42.

4. Kemp JP, Smith PM, Pyle A, et al. Nuclear factors involved in mitochondrial transla- 
tion cause a subgroup of combined respiratory chain deficiency. Brain 2011;134: 18395.

5. Smits P, Saada A, Wortmann SB, Heister AJ, Brink M, Pfundt R, et al. Mutation in mitochondrial ribosomal protein MRPS22 leads to Cornelia de Lange-like phenotype, brain abnormalities and hypertrophic cardiomyopathy. Eur J Hum Genet 2011; 19:394-9.

6. Palmieri L, Alberio S, Pisano I, et al. Complete loss-of-function of the heart/muscle-specific adenine nucleotide translocator is associated with mitochondrial myopathy and cardiomyopathy. Hum Mol Genet 2005;14:3079-88.

7. Suomalainen A, Paetau A, Leinonen $\mathrm{H}$, et al. Inherited idiopathic dilated cardiomyopathy with multiple deletions of mitochondrial DNA. Lancet 1992;340:1319-20.

8. Azevedo 0, Vilarinho L, Almeida F, et al. Cardiomyopathy and kidney disease in a patient with maternally inherited diabetes and deafness caused by the $3243 \mathrm{~A}>\mathrm{G}$ mutation of mitochondrial DNA. Cardiology 2010;115:71-4.

9. Chinnery PF, Johnson MA, Wardell TM, et al. The epidemiology of pathogenic mitochondrial DNA mutations. Ann Neurol 2000;48:188-93.

10. Hsu PC, Chu CS, Lin TH, et al. Adult-onset hypertrophic cardiomyopathy manifested as initial major presentation of mitochondrial disease with A-to-G 3243 tRNA (Leu(UUR)) point mutation. Int J Cardiol 2007;129:441-3.

11. Takahashi N, Shimada T, Ishibashi Y, et al. Marked left ventricular hypertrophy in a patient with mitochondrial myopathy, encephalopathy, lactic acidosis, and strokelike episodes. Int J Cardiol 2008;129:e77-80.

12. Tsujita Y, Kunitomo T, Fujii M, et al. A surviving case of mitochondrial cardiomyopathy diagnosed from the symptoms of multiple organ dysfunction syndrome. Int $\mathbf{J}$ Cardiol 2008;128:e43-5.

13. Majamaa-Voltti K, Peuhkurinen K, Kortelainen ML, et al. Cardiac abnormalities in patients with mitochondrial DNA mutation 3243A $>$ G. BMC Cardiovasc Disord 2002;2:12.

14. Goto Y, Nonaka I, Horai S. A mutation in the tRNA(Leu)(UUR) gene associated with the MELAS subgroup of mitochondrial encephalomyopathies. Nature 1990;348:651-3.

15. van den Ouweland JM, Lemkes HH, Ruitenbeek W, et al. Mutation in mitochondrial tRNA(Leu)(UUR) gene in a large pedigree with maternally transmitted type II diabetes mellitus and deafness. Nat Genet 1992;1:368-71.

16. Majamaa K, Moilanen JS, Uimonen S, et al. Epidemiology of A3243G, the mutation for mitochondrial encephalomyopathy, lactic acidosis, and strokelike episodes: prevalence of the mutation in an adult population. Am J Hum Genet 1998;63:447-54.

17. Manwaring N, Jones MM, Wang JJ, et al. Population prevalence of the MELAS A3243G mutation. Mitochondrion 2007;7: 230-3.

18. Pietiläinen KH, Naukkarinen J, Rissanen A, et al. Global transcript profiles of fat in monozygotic twins discordant for BMI: pathways behind acquired obesity. PLoS Med 2008;5:e51.

19. Suomalainen A, Syvänen AC. Quantitative analysis of human DNA sequences by PCR and solid-phase minisequencing. Mol Biotechnol 2000;15:123-31.

20. Klement P, Nijtmans LG, Van den Bogert C, Houstek J. Analysis of oxidative phosphorylation complexes in cultured human fibroblasts and amniocytes by blue-native-electrophoresis using mitoplasts isolated with the help of digitonin. Anal Biochem 1995;231:218-24.

21. Wittig I, Braun HP, Schagger H. Blue native PAGE. Nat Protoc 2006;1:418-28.

22. Zerbetto E, Vergani L, Dabbeni-Sala F. Quantification of muscle mitochondrial oxidative phosphorylation enzymes via histochemical staining of blue native polyacrylamide gels. Electrophoresis 1997;18:205964.

23. Kirino Y, Goto Y, Campos Y, et al. Specific correlation between the wobble modification deficiency in mutant tRNAs and the clinical features of a human mitochondrial disease. Proc Natl Acad Sci U S A 2005;102:7127-32.

24. Rahman S. Mitochondrial disease and epilepsy. Dev Med Child Neurol 2012;5: 397406.

25. Rahman S, Poulton J, Marchington D, Suomalainen A. Decrease of $3243 \mathrm{~A} \longrightarrow \mathrm{G}$ mtDNA mutation from blood in MELAS syndrome: a longitudinal study. Am J Hum Genet 2001;68:238-40.

26. Alfakih K, Reid S, Jones T, Sivananthan M. Assessment of ventricular function and mass by cardiac magnetic resonance imaging. Eur Radiol 2004;14:1813-22.

27. Clay S, Alfakih K, Radjenovic A, et al. Normal range of human left ventricular volumes and mass using steady state free precession MRI in the radial long axis orientation. MAGMA 2006;19:41-5.

28. Campos Y, Garcia A, del Hoyo P, et al. Two pathogenic mutations in the mitochondrial DNA tRNA Leu(UUR) gene (T3258C and A3280G) resulting in variable clinical phenotypes. Neuromuscul Disord 2003;13:41620.

29. Sproule DM, Kaufmann P. Mitochondrial encephalopathy, lactic acidosis, and stroke- like episodes: basic concepts, clinical phenotype, and therapeutic management of MELAS syndrome. Ann N Y Acad Sci 2008;1142:133-58.

30. Jefferies JL, Towbin JA. Dilated cardiomyopathy. Lancet 2010;375:752-62.

31. Wahbi K, Larue S, Jardel C, et al. Cardiac involvement is frequent in patients with the m.8344A>G mutation of mitochondrial DNA. Neurology 2010;74:674-7.

32. Mahrholdt H, Wagner A, Judd RM, et al. Delayed enhancement cardiovascular magnetic resonance assessment of nonischaemic cardiomyopathies. Eur Heart J 2005;26:1461-74.

33. Cummings KW, Bhalla S, Javidan-Nejad C, et al. A pattern-based approach to assessment of delayed enhancement in nonischemic cardiomyopathy at MR imaging. Radiographics 2009;29:89-103.

34. Sasarman F, Antonicka H, Shoubridge EA. The A3243G tRNALeu(UUR) MELAS mutation causes amino acid misincorporation and a combined respiratory chain assembly defect partially suppressed by overexpression of EFTu and EFG2. Hum Mol Genet 2008;17:3697-707.

35. Mariotti C, Tiranti V, Carrara F, et al. Defective respiratory capacity and mitochondrial protein synthesis in transformant cybrids harboring the tRNA(Leu(UUR)) mutation associated with maternally inherited myopathy and cardiomyopathy. J Clin Invest 1994;93: 1102-7.

36. Turner LF, Kaddoura S, Harrington D, et al. Mitochondrial DNA in idiopathic cardiomyopathy. Eur Heart J 1998;19:1725-9.

37. Zeviani M, Mariotti C, Antozzi C, et al. OXPHOS defects and mitochondrial DNA mutations in cardiomyopathy. Muscle Nerve 1995;3:S170-4.

38. Grasso M, Diegoli M, Brega A, et al. The mitochondrial DNA mutation T12297C affects a highly conserved nucleotide of tRNA(Leu(CUN)) and is associated with dilated cardiomyopathy. Eur J Hum Genet 2001; 9:311-5.

39. Merante F, Myint T, Tein I, et al. An additional mitochondrial tRNA(lle) point mutation (A-to-G at nucleotide 4295) causing hypertrophic cardiomyopathy. Hum Mutat 1996; 8:216-22.

40. Merante F, Tein I, Benson L, Robinson BH. Maternally inherited hypertrophic cardiomyopathy due to a novel T-to-C transition at nucleotide 9997 in the mitochondrial tRNA(glycine) gene. Am J Hum Genet 1994; 55:437-46. 